\title{
OPERATORS AND INITIAL-VALUE PROBLEMS
}

\author{
R. M. REDHEFFER
}

1. Introduction. This paper gives representation theorems for certain two-parameter families of linear operators $\phi=\phi_{a b}, b>a$, where the domain and range of each operator $\phi$ is a subset of the set of all complex-valued functions $f(x),-\infty<x<\infty$. Linearity means, of course, that

$$
\phi(p f+q g)=p \phi f+q \phi g
$$

for any complex numbers $p, q$ and any functions $f, g$ in the domain of $\phi$.

The operators are said to be bounded pointwise by $M_{a b}$ if $\phi_{a b} f$ exists whenever $f \in L^{2}$, and satisfies

$$
\left|\boldsymbol{\phi}_{a b} f\right|^{2} \leqq M_{a b} \int|f|^{2}
$$

for some constants $M_{a b}$. They are said to be bounded in mean by $N_{a b}$ if $\phi_{a b} f$ is measurable and

$$
\int\left|\phi_{a b} f\right|^{2} \leqq N_{a b}\left(\int|f|\right)^{2}
$$

whenever simultaneously $f \in L, f \in L^{2}$. The class is closed if

$$
\phi_{a b} \phi_{b c} f=\phi_{a c} f
$$

whenever $a<b<c$ and $f \in L, f \in L^{2}$.

A representation theorem for linear operators $\phi_{a b}$ which are bounded pointwise, bounded in mean, and closed, is given in Theorem I. Further specialization of the operator class leads to the representations given by Theorems II, III, and IV.

In these theorems, and throughout this paper, the symbol $T$ stands for Fourier transforms,

$$
T f=(2 \pi)^{-1 / 2} \underset{m \rightarrow \infty}{\operatorname{lim.m}} \int_{-m}^{m} f(x) e^{-i u x} d x,
$$

and $T^{-1}$ for the same with $i$ in place of $-i$. By the Plancherel theorem $T T^{-1}=1$ in the mean-square sense. In case $f \in L$ the li.m. in (5) is to be replaced by lim, which then exists for all real $u$. We also use the

Presented to the Society, May 3, 1952; received by the editors October 23, 1950, November 6, 1950 and, in revised form, November 13, 1952. 
* notation for convolution,

$$
f(y) * g(y)=\int_{-\infty}^{\infty} f\left(y_{1}\right) g\left(y-y_{1}\right) d y_{1}
$$

The operators $\phi_{a b}$ considered here originate in specific problems of classical mathematics and physics. For illustration and orientation, some of these specific problems are briefly mentioned in \$\$2-5, where we also discuss the physical background of the assumptions defining our operator class. Since this collateral discussion is not essential to the theorems or their proofs, the latter are presented independently in $8 \$ 6-8,10-12$.

\section{BACKGROUND AND CONTEXT}

2. Examples. The partial differential equation associated with certain types of initial-value problems enables one to compute a disturbance at a given point, if the disturbance is known over a suitable surface. In antenna theory, for example, a typical problem for the scalar potential in the $(x, y)$ plane is to solve $U_{x x}+U_{y y}+k^{2} U=0$, $x>0$, subject to $U(0, y)=f(y)$, a known function. The trial solution $\exp (a x+b y)$ leads to

$$
e^{i x\left(k^{2}-u^{2}\right)^{1 / 2}+i u y}
$$

when we ask that the "small-angle" propagation, $|u|<k$, occur without attenuation. The positive branch of the radical is taken to give attenuation for $|u|>k$, the modes beyond cutoff in waveguide terminology. Forming a general linear combination of expressions (7) suggests that perhaps

$$
U(x, y)=(2 \pi)^{-1 / 2} \int_{-\infty}^{\infty} F(u) e^{i x\left(k^{2}-u^{2}\right)^{1 / 2}+i u y} d u
$$

for a suitable function $F(u)$.

With $F=F(u)$ and with

$$
\log \Lambda=i\left(k^{2}-u^{2}\right)^{1 / 2},
$$

equation (8) may be written

$$
U(x, y)=T^{-1} F \Lambda^{x} .
$$

Putting $x=0$ in (10) gives $U(0, y)=f(y)=T^{-1} F$, so that $F=T f$ when $f \in L^{2}$. Thus it is that one solution ${ }^{1}$ of the problem is

1 A longer derivation of much the same result is given in [1]. Actually it is well known that (11) arises in connection with linear partial differential equations having constant coefficients. See [2], for example. 


$$
U(x, y)=T^{-1} \Lambda^{x} T U(0, y)
$$

where $T$ is the Fourier transform in the $L^{2}$ sense and $T^{-1}$ the inverse transform in the $L$ sense.

As another example, let it be required to find the temperature $U(x, y)$ at time $x$ in a uniform medium, given the initial temperature $U(0, y)$. The foregoing procedure applied to the equation $U_{x}=k U_{y y}$ yields (11) with

$$
\log \Lambda=-k u^{2}
$$

when we require suitable behavior for large $x$ and $y$. Similarly, the problem of finding a harmonic function $U(x, y)$ which reduces to $f(y)$ $\in L^{2}$ when $x=0$ gives (11) as one solution, with

$$
\log \Lambda=-|u|
$$

when suitable regularity conditions are postulated.

3. Convolution. Suppose given a function $f(z)=f(x+i y)=U(x, y)$ analytic in the right half-plane, and satisfying there $\max |f(z)| \rightarrow 0$ on $|z|=r$ as $r \rightarrow \infty$. Cauchy's integral theorem gives

$$
U(x, y)=\lambda_{x}(y) * U(0, y)
$$

where $\lambda_{x}(y)=(2 \pi)^{-1 / 2}(x+i y)^{-1}$. Equation (14) exemplifies "propagation," in that $f(z)$ is determined at the point $z$ from its values on the line $x=0$. Introducing the convolution theorem

$$
T(f * g)=(T f)(T g)
$$

we find that (14) takes the form (11), where

$$
\begin{aligned}
\log \Lambda & =u & & (u<0), \\
\Lambda & =0 & & (u>0) .
\end{aligned}
$$

Conversely, if we define $\lambda_{x}(y)$ by

$$
\lambda_{x}(y)=T^{-1}[\Lambda(u)]^{x},
$$

so that $\Lambda^{x}=T \lambda_{x}(y)$, then (11) becomes (14). In the context of differential equations (14) corresponds to the representation by singular solutions or source functions. Since $T^{-1} \exp \left(-k x u^{2}\right)=(2 k x)^{-1 / 2}$ $\exp \left(-y^{2} / 2 k x\right)$, for example, (14) leads to the familiar form for the above problem in heat conduction.

4. Discussion. When a function $f(b, y)$ is determined for $b>a$ by the initial values $f(a, y)$, one may think of the function as a disturbance or field which is propagated from $a$ to $b$. The foregoing 
examples illustrate this meaning for the term propagation, and lead the way to a more abstract formulation. As in the examples, we suppose that the problem is one-dimensional, and that the "surface" on which the disturbance is known is the $y$ axis. Again as in the examples, the disturbance is supposed to depend on the values alone; it is not necessary to know the normal derivatives. An extension to arbitrary surfaces, to higher dimensions, and to more detailed dependence on the initial conditions is desirable but is not attempted.2

Within these limitations, our problem is to derive the form of a "propagation principle" from general considerations, without reference to the differential equation which the disturbance may satisfy. Answers are given to such questions as the following: Is it a consequence of the nature of propagation that the disturbance propagate by an operator of form $T^{-1} \lambda(x, u) T$ ? If so, is it necessary that the propagation variable $x$ appear as an exponent, $\lambda(x, u)=[\Lambda(u)]^{x}$ ? In that case is it necessary that $\log \Lambda(u)$ be an algebraic function of $u$ ? What are the analogues of these properties for propagation in a nonuniform medium? The relevance of the discussion to certain problems in physics has already been suggested; the connection with mathematical fields of current interest is mentioned at appropriate points in the sequel. ${ }^{3}$

Instead of obtaining the disturbance (that is, the functional values) at a single point, it is more convenient to think of the disturbance on a second surface similar to the one on which the data are given. This viewpoint leads to a formulation in terms of operators. In Cartesian coordinates $(x, y)$, then, let the disturbance be $f(y), g(y)$, on the lines $x=a, x=b>a$ respectively. Thus, $f(y)$ plays the role of $U(a, y)$, and $g(y)$ of $U(b, y)$, in the foregoing discussion. Define $\phi=\phi_{a b}$ as the operator which transforms $f$ into $g$, so that $g=\phi f$. We remark, first, that $\phi$ may depend on the intervening medium, and second, that mere introduction of $\phi$ implies that the disturbance is propagated, in the sense of this article. A similar construction of a family of operators from a family of functions is given in [2].

5. Formulation of the assumptions. Physically, $\int|f|^{2} d x$ is associated with the power in the disturbance, at least in the sense that the one is finite whenever the other is. By concentrating the initial disturbance $f$ more and more, one can make the amplitude $\left|f\left(x_{0}\right)\right|$ at a particular point $x_{0}$ arbitrarily large, and yet have $\int|f|^{2}<1$. Thus is

\footnotetext{
2 It is probable that such an extension could be based easily on the analysis in [2], where indeed certain aspects of the problem are already attacked.

3 The author is indebted to the referee for many of the items cited.
} 
obtained the so-called point source of physical optics or the impulse function of circuit analysis. Yet even in this case $\phi_{a b} f$ remains bounded for $b>a$ : one cannot propagate a disturbance of large magnitude through a positive distance, unless there is large power. These considerations suggest the condition of pointwise boundedness (2). A similar line of thought concerning the power rather than the amplitude of $\phi f$ leads to the condition of mean boundedness (3).

To discuss the closure condition (4), let $h(y)$ be the disturbance on the plane $x=c>b$, retaining $f$ and $g$ as before on the planes $x=a$, $x=b>a$. If the functions concerned belong to $L^{2}$, then one can compute $h$ from $g$ as as $h=\phi_{b c} g$, or one can find $h$ directly from $f$ as $\phi_{a c} f$. Hence it is relevant to consider operators satisfying $\phi_{a c} f=\phi_{b c} \phi_{a b} f$ for a suitable class $f$; and this is (4). When the parameters $a, b$ refer to time, the closure property is the "major premise" of Huygens' principle according to Hadamard (cf. $[2 ; 3]$ ). There is then no question of nonuniformity of the propagating medium; the two-parameter family $\phi_{a b}$ becomes a one-parameter family $\phi_{t}, t=b-a$; and the closure property is the principle of physical determinism. The operators are then essentially Wiener's "operators of the closed cycle," and the semigroup which arises has been considered in [2]. The closure condition for the two-parameter case $\phi_{a b}$ is equivalent to the ChapmanKolmogoroff equation of probability theory (cf. item iv of Theorem I).

\section{THE REPRESENTATION THEOREMS}

6. The general case. It is thought that the preceding explanations make clear the relevance of the theorem, which is now stated without further reference to its background:

THEOREM I. Suppose given a class of operators $\left\{\phi_{a b}\right\}$ which are linear, bounded pointwise by $M_{a b}$, bounded in mean $N_{a b}$, and closed. Then it follows that:

$$
\text { i. } \phi_{a b} f=\int \lambda_{a b}(x, y) f(x) d x \text {, }
$$
where

$$
\begin{aligned}
& \text { ii. } \int\left|\lambda_{a b}(x, y)\right|^{2} d x \leqq M_{a b} ; \\
& \text { iii. } \int\left|\lambda_{a b}(x, y)\right|^{2} d y \leqq N_{a b} \text { for almost all } x \text {; }
\end{aligned}
$$

iv. $\int \lambda_{a b}(x, y) \lambda_{b c}(y, z) d y=\lambda_{a c}(x, z)$ for almost all $x$. Also the conditions of the hypothesis are independent, and they are implied by i-iv.

For any fixed values of $y, a$, and $b$ the operator $\phi$ is a linear functional on the Hilbert space $L^{2}(-\infty, \infty)$, and it is bounded in the appropriate sense by our condition of pointwise boundedness. Hence $i$ 
and ii of the theorem are immediate, by a theorem of F. Riesz.4

If $S_{\delta}(x, y)=(1 / 2 \delta) \int_{x-\delta}^{x+\delta} \lambda(t, y) d t$, then $\lim _{\delta \rightarrow 0} S_{\delta}=\lambda(x, y)$ for almost all $x$ and any fixed $y$. Let $x$ be a value for which the equality holds and define $f_{\delta}(t)=1 / 2 \delta$ for $|t-x| \leqq \delta, f_{\delta}=0$ otherwise. Then $\phi f_{\delta}=S_{\delta}$, so that boundedness in mean gives $\int_{-m}^{m}\left|S_{\delta}(x, y)\right|^{2} d y \leqq N$ for every $m>\delta$. By the theorem of Fatou we have then $\int_{-m}^{m}|\lambda(x, y)|^{2} d y \leqq N$, and from this $\int_{-\infty}^{\infty}|\lambda(x, y)|^{2} d y \leqq N$, giving iii.

Now it is known that $i$ plus closure in our sense leads to iv, but here we give a simple proof making use of iii. Closure leads to

$$
\begin{aligned}
\lim _{n \rightarrow \infty} \int_{-n}^{n}\left\{\int_{-m}^{m} f(x) \lambda_{a b}(x, y) d x\right\} \lambda_{b c}(y, z) d y & \\
& =\int_{-m}^{m} f(x) \lambda_{a c}(x, z) d x
\end{aligned}
$$

if we choose $f \in L^{2}, f=0$ for $|x|>m$. The theorem of Fubini applies to (18), as we see by using the Schwarz inequality on the inner integral, and therefore

$$
\lim _{n \rightarrow \infty} \int_{-m}^{m} f(x)\left\{\int_{-n}^{n} \lambda_{a b}(x, y) \lambda_{b c}(y, z) d y-\lambda_{a c}(x, z)\right\} d x=0
$$

for all $f(x) \in L^{2}$ on $(-m, m)$. Together with iii, the Schwarz inequality shows $\int\left|\lambda_{a b}(x, y) \lambda_{b c}(y, z)\right| d y \leqq M N$ uniformly in $x, z$, so that one may interchange lim and $\int$ in (19). By choosing $f(x)$ to be the conjugate of the resulting expression in braces one obtains iv.

To prove independence we give examples with $\boldsymbol{\phi}_{a b}=\phi$ independent of $a$ and $b$. Let $\phi f(x)=g(y)$ for convenience of verbalization. If $\phi$ is defined by $g(0)=\left[\int_{-1 / 2}^{1 / 2}|f|^{2} d x\right]^{1 / 2}, g(y)=0$ for $y \neq 0$, then $\phi$ satisfies all but linearity. If $g(0)=f(0), g(y)=0$ for $y \neq 0$, then $\phi$ satisfies all but pointwise boundedness. If $g(y)=\int_{-1 / 2}^{1 / 2} f(x) d x$, then $\phi$ satisfies all but mean boundedness, and if $g(0)=\int_{-1}^{1} f(x) d x, g(y)=0$ for $y \neq 0$, then $\phi$ satisfies all but closure.

To show that i, ii, iii, and iv together imply the hypothesis, note that $i$ and ii give linearity and pointwise boundedness by $M_{a b}$. For mean boundedness write $\int|\phi f|^{2}$ in the form $\int_{-m}^{m} \int_{-n}^{n} \int_{-n}^{n} \lambda(x, y) f(x) \lambda^{*}(t, y) f^{*}(t) d x d t d y$. Integrating with respect to $y$ gives $\int_{-m}^{m}|\lambda(x, y)|\left|\lambda^{*}(t, y)\right| d y \leqq N^{1 / 2} N^{1 / 2}$ by the Schwarz inequality, so that the triple integral is absolutely convergent, and dominated by $N\left(\int|f|\right)^{2}$. Since these bounds are independent of $m$ and $n$ we have established mean boundedness by $N_{a b}$.

- The author is indebted to Alan Kay for this proof of $i$ and ii, which is shorter than the proof originally given. 
Closure leads us to consider

$$
\int_{-\infty}^{\infty}\left(\int_{-n}^{n} \lambda_{a b}(x, y) \lambda_{b c}(y, z) d y\right) f(x) d x
$$

after changing the order of integration. The inversion is justified by ii and iii applied to the inner integral. Letting $n \rightarrow \infty$ and using iv gives closure, the Lebesgue theorem on bounded convergence being applicable here.

7. Uniformity of the medium in two directions. If the medium is uniform in $y$, then the discussion can be carried further. In terms of the operator $\phi$, uniformity in $y$ means that

$$
\phi f(x-c)=g(y-c)
$$

whenever $\phi f=g$, for every real constant $c$.

If the constants of the medium are finite, then an electromagnetic disturbance never dies out altogether, after propagation through a finite distance. In the framework of the present discussion, this means that

$$
\phi f \not \equiv 0 \text { if } \int|f|^{2}>0,
$$

and operators satisfying such a condition are termed nonvanishing.

THEOREM II. Let $\left\{\phi_{a b}\right\}$ be a class of operators for a medium uniform in $y$, which are linear, bounded pointwise by $M_{a b}$, closed, nonvanishing, and such that $\phi_{0 a} f$ is a measurable function of $a$. Then

i. $\phi_{a b}=T^{-1} \Lambda_{a b} T$, where $T$ denotes the Fourier transform in the $L^{2}$ sense, $T^{-1}$ the inverse transform in the $L$ sense. Also

ii. $\Lambda_{a b}(u)=\exp \int_{a}^{b} d_{x} \alpha(x, u)$ for some function $\alpha$ of bounded variation in $x$ on every finite interval, and

iii. $\int\left|\Lambda_{a b}(u)\right|^{2} d u \leqq M_{i a b}$.

Conversely, i, ii, iii imply the hypothesis.

For proof, note that again we have $\mathrm{i}$, ii of Theorem I, and uniformity gives $\lambda(x, y) f(x-c) d x=\lambda \lambda(x, y-c) f(x) d x$, so that $\lambda(x+c, y)$ $=\lambda(x, y-c)$ for almost all $x$. This last follows by letting $t=x-c$ in the first integral, taking $f(x)=e^{-x^{2}} e^{i u x}$, and using uniqueness for fixed $y$. Replacing $y$ by $y+c$ and then picking $c=-x$ gives $\lambda(0, y-x)$ $=\lambda(x, y)$ so that for almost all $x$ (and hence always in $\phi f$ ) one may write $\lambda(x, y)=\lambda(y-x)$.

Since $\lambda(x, y) \in L^{2}$ in $x$, we have $\lambda(x) \in L^{2}$. The operator $\phi f$ is now the convolution of $f$ and $\lambda$, so that it is natural to introduce the Fourier 
transform (5). With $T \lambda=\Lambda=\Lambda_{a b}(u)$ we have $T(\phi f)=\Lambda \cdot(T f)$ by the convolution theorem, whence i follows, since $\Lambda_{a b} T f \in L$. Also the earlier results on $\lambda(x, t)$ combine with the Parseval equality to give iii.

The closure condition is now $\left(T^{-1} \Lambda_{b c} T\right)\left(T^{-1} \Lambda_{a b} T\right) f=\left(T^{-1} \Lambda_{a c} T\right) f$ so that $\Lambda_{b c} \Lambda_{a b}=\Lambda_{a c}$ almost everywhere, again by uniqueness. Our aim is to redefine $\Lambda$ in such a way that this holds everywhere.

First, if $\Lambda_{a b}=0$ in a set $E_{a b}$ then $m\left(E_{a b}\right)=0$. Otherwise the function $F=e^{-x^{2}}$ in $E, F=0$ in $C E$ yields a function $f=T^{-1} F$, for which the positive character of $\phi_{a b}$ is violated. Next, define $\Lambda_{0 a}=1$ in $E_{0 a}$, and $\Lambda=1$ also at points where $\Lambda$ is still undefined. From $\Lambda_{0 b} \Lambda_{b c}=\Lambda_{0 c}$, which holds almost everywhere even with the new definition, one may define $\Lambda_{b c}=\Lambda_{0 c} / \Lambda_{0 b}$; the right side is well determined. Then $\Lambda_{a b} \Lambda_{b c}$ $=\left(\Lambda_{0 b} / \Lambda_{0 a}\right)\left(\Lambda_{0 c} / \Lambda_{0 b}\right)=\Lambda_{0 c} / \Lambda_{0 a}=\Lambda_{a c}$ everywhere; $\Lambda \neq 0$ everywhere; and $\mathrm{i}$ is still valid.

Introducing $A_{a b}=\log \Lambda_{a b}$ we see that $A_{a b}$ is a measurable and additive interval function, so that $A_{a b}=\int_{a}^{b} d \alpha(x)$ for each $u$, by another theorem of F. Riesz; and the result ii is established. The converse presents no difficulty.

8. Homogeneous medium. In case there is uniformity in $x$, then $\phi_{a b}$ depends only on $b-a$, the distance through which the disturbance is propagated; hence uniformity in $x$ means

$$
\phi_{a, b}=\phi_{a+c, b+c}
$$

for all real $c$. Here $\phi_{a b}=\phi_{a, b}$.

If the medium parameters are finite, then the disturbance varies continuously from point to point, hence $\phi_{a b} f$ is continuous in $a$ and $b$. Such an assumption simplifies the results for the homogeneous case.

THEOREM III. Let $\phi_{a b}$ be a class of operators for a homogeneous medium, which are linear, bounded pointwise by $M_{a b}$, closed, and such that $\phi_{0 a}$ is a continuous function of $a$. Then it follows that

i. $\phi_{a b}=T^{-1} \Lambda^{b-a} T$, where $T$ denotes the Fourier transform in the $L^{2}$ sense, $T^{-1}$ the inverse transform in the $L$ sense. Also

ii. $\int|\Lambda(u)|^{2(b-a)} d u \leqq M_{a b}$ for all $b>a$.

Conversely, all operators satisfying i, ii satisfy the conditions of the hypothesis.

As before $\phi_{a b}=T^{-1} \Lambda_{a b} T$, and closure gives $\Lambda_{p} \Lambda_{q}=\Lambda_{p+q}$, if we write $\Lambda_{a, a+p}=\Lambda_{p}$ to introduce $x$ uniformity. The equation holds for almost all $u$ and for all positive $p, q$; if it held everywhere and if $\Lambda_{p}$ were continuous in $p$, then we should have $\Lambda_{p}=\left(\Lambda_{1}\right)^{p}$. Following through the proof of this well known result, one obtains first $\left(\Lambda_{p}\right)^{n}=\Lambda_{n p}$ for 
$n=1,2,3, \cdots$ and almost all $u$. Redefine $\Lambda$ so this holds for all $u$ and all rational $p$, using the fact that a countable sum of sets having measure zero again has measure zero. The choice $p=m / n$ shows $\Lambda_{p}=\left(\Lambda_{1}\right)^{p}$ for all rational $p>0$. Finally, continuity shows $\phi_{0, a}$ $f=\lim T^{-1}(\Lambda)^{p} T f$ if $p \rightarrow a$ through rationals; and therefore the definition $\Lambda_{a}=\left(\Lambda_{1}\right)^{1 / a}$ leaves $\phi$ unchanged. This uses the Schwarz inequality, bounded convergence, and the result ii, which is obtained as in the previous theorem. The proof of III is now complete, except for the trivial converse.

9. Extension. The function $\phi f$ may exist in the sense of Theorem I and yet not in the sense of II or III. For electromagnetic theory this is the case even with such simple illuminations as $f=1$. More generally, introduction of $T$ automatically rules out all infinite plane waves, although for them $\phi f$ always makes sense. This generality of $\phi$ (as opposed to $T$ ) is one reason why such devices as the Dirac delta function consistently give correct answers in diffraction problems.

How shall we define $\phi$ so that the class of admissible functions is restricted only insofar as is physically essential? Imagine the plane $x=a$ covered by a symmetrically-located aperture of width $2 m$ which gradually widens. Then we define the field without aperture to be the limit of the field with aperture as the width becomes infinite. Mathematically what this does is to introduce $f_{m}=f$ for $|y| \leqq m, f_{m}=0$ for $|y|>m$, and to define $\phi f=\lim _{m \rightarrow \infty} \phi f_{m}$. Thus one can often leave the classes $L$ or $L^{2}$, and yet have a well-determined value for $\phi f$. In the notation of I we are simply taking the Cauchy principal value for the infinite integrals, but in II and III the procedure is less trivial.

To illustrate this notion of extension we consider the special case presented by physical optics. Let it be supposed that the (one-dimensional) propagation of electromagnetic radiation in a uniform medium satisfies the hypothesis of Theorem III. To determine the arbitrary function $\Lambda$, which is present even for uniform media, recall that propagation of plane waves through a distance $d$ corresponds to multiplication by $e^{i k d}$. Using the conclusion of Theorem III, we shall see that the propagation operator $\phi_{a b}$ is completely determined by this condition alone. Since $e^{i k d}$ is not in $L$ or $L^{2}$, the discussion requires the method of the foregoing paragraph.

Given a wave in direction $\theta=\sin ^{-1} u / k$ one has $f(x)=e^{i u x}, \phi_{0 a} f$ $=\exp \left(i a\left(k^{2}-u^{2}\right)^{1 / 2}+i u y\right)$ by inspection. On the other hand $\phi_{0 a} f$ $=\lim T^{-1} \Lambda^{a} T f_{m}$ as described above. An elementary calculation gives $T f_{m}$, so that

$$
\phi_{0 a} f=\lim _{m \rightarrow \infty} \frac{1}{\pi} \int_{-\infty}^{\infty} e^{i x \nu}[\Lambda(x)]^{a} \frac{\sin (u-x) m}{u-x} d x .
$$


Since $\Lambda^{a / 2} \in L^{2}$ we have $\Lambda^{a} \in L$, and therefore the limit is $e^{i u v}[\Lambda(u)]^{a}$ almost everywhere. Comparison of the two forms $\phi_{0 a}$ yields (9) and (11). The other examples of the introduction can be similarly dealt with; the $\Lambda(u)$ appropriate to the given disturbance is obtained, as here, by specifying the propagation of a special class of functions. This use of special functions is analogous to the use of a trial solution $e^{a x+b y}$ in the heuristic treatment first discussed.

\section{Partial differential EQUATIONS}

10. The result. Explicit introduction of the wave equation has an air of too great specialization, and we do not pursue the subject. But one may quite properly suppose that the disturbance satisfies a differential equation of some kind. Even with no specification concerning the form of the equation, such an assumption gives a notable restriction on the operator class.

TheOREM IV. Let $U(x, y)=T^{-1} F \Lambda^{x}=(2 \pi)^{-1 / 2} \int_{-\infty}^{\infty} e^{i t y}[\Lambda(t)]^{x} F(t) d t$, where $\Lambda(t)$ is measurable. Suppose $U$ satisfies one and the same partial differential equation for all $F$ giving sufficiently strong convergence of the integral. Then

i. $\log \Lambda(t)$ is equal almost everywhere to one and the same algebraic function of $t$, except when $\Lambda=0$ or when $\Lambda$ is undefined.

ii. For all $x$ and $y$, and all $F$ giving good convergence, the functions $U(x, y)$ are solutions of one and the same linear homogeneous partial differential equation with constant coefficients.

In the hypothesis, when we say that $U$ satisfies a differential equation we mean that there is a function $\psi$ for which

$$
\psi\left(U, U_{x}, U_{y}, U_{x x}, U_{y y}, \cdots ; x, y\right)=0
$$

at some point $\left(x_{0}, y_{0}\right)$ where $\psi$ is not identically zero. Also there are only a finite number of variables in $\psi$.

11. A lemma. The partial differential equation (24) may be written

$$
\psi(V, X)=0
$$

where $X=(x, y)$ and where the components of $V$ are $U$ and certain of its partial derivatives. Although $\psi \neq \equiv$, for certain values of $\boldsymbol{X}$ it may happen that $\psi \equiv 0$ in $V$. The set in $X$ where this occurs is termed the zero set of $\psi$. For example the equation $(\log x) U_{x}+y \sin \left(U_{y y}\right)=x y U_{y}^{2}$ has $X=(x, y), V=\left(U_{x}, U_{y}, U_{y y}\right)$; the zero set is the point $x=1, y=0$. The equation $(y-x)^{2} Y_{y y}-\sin (y-x) \log U=0$ has the whole line $y=x$ as zero set. 
LEMMA. Let a family of functions $\left\{U_{p}(X)\right\}$ satisfy a partial differential equation $\psi(V, X)=0$ for a certain set $S$ in $X$, and for all $p$. Suppose further that $a U_{\alpha}+b U_{\beta}$ is in the family $\left\{U_{p}\right\}$ whenever $U_{\alpha}$ and $U_{\beta}$ are, $a$ and $b$ being any constants. Then the family satisfies one and the same linear homogeneous partial differential equation at every point of $S$ which is not in the zero set of $\psi$.

To prove this, pick a fixed vector $X_{0}$ not in the zero set but in the set $S$ where the equation holds. At $X=X_{0}$ each $p$ gives rise to a solution $U_{p}$, which in turn gives rise to a derivative vector $V_{p}$. It is easy to see that linear closure of $\left\{U_{p}\right\}$ implies linear closure of $\left\{V_{p}\right\}$. Assuming that each $V_{p}$ has, say, $m$ components, we assert that as $p$ varies the vectors $V_{p}$ cannot span the $m$-dimensional space.

For suppose they do. Then one can pick a basis $V_{j} \in\left\{V_{p}\right\}$ $(j=1,2, \cdots, m)$ such that any vector $V=\sum a_{j} V_{j}$ for suitable constants $a_{j}$. Being a linear combination, $V$ must be in the set $\left\{V_{p}\right\}$; hence $\psi\left(V, X_{0}\right)=0$; and therefore $X_{0}$ is in the zero set, contrary to hypothesis.

Since $\left\{V_{p}\right\}$ does not span the $m$-dimensional space, there is a nonzero vector $\boldsymbol{A}$ (depending on $\boldsymbol{X}_{0}$ ) such that $\boldsymbol{A} \cdot \boldsymbol{V}_{p}=0$ for all $p$. Writing out in terms of components and remembering $A=A(X)$ we obtain the lemma.

12. Proof of the theorem. Returning to the proof of IV, we begin by writing

$$
\begin{aligned}
\Lambda(t) & =e^{s(t)} & (t \text { in } E), \\
F & =e^{-|s|^{2}-t^{2} p(t)} & (t \text { in } E), \\
F & =0 & (t \text { in } C E),
\end{aligned}
$$

where $p$ is any continuous bounded function which vanishes outside of a finite interval. Here $E$ is the set of values $t$ at which $\Lambda$ is defined and $\Lambda \neq 0$. Since the integral with such an $F$ is sure to converge uniformly and absolutely, and since the same is true for derivatives $U_{x} \cdots$ of any order, the class (26) will be considered to give "sufficiently strong" convergence. There is no need for more general $F$ in the proof.

Since $a p_{1}+b p_{2}$ is bounded, continuous, and vanishing when $p_{1}$ and $p_{2}$ are, it is seen that the closure condition of the lemma is satisfied for the family $\left\{U_{p}\right\}$ which arises from the various choices of $p$. Pick $X_{0}=\left(x_{0}, y_{0}\right)$ in $S$ but not in the zero set to find

$$
\sum a_{k j}(\partial / \partial y)^{k}(\partial / \partial x)^{j} U=0
$$

at the point $\boldsymbol{X}_{0}$. By the lemma this holds true for all choices of $p$. 
The class $F$ has been so chosen that we can differentiate any number of times under the integral sign, to replace (28) by

$$
\int_{E}\left(\sum a_{j k} s^{j}(i t)^{k} e^{-|\imath|^{2}-t^{2}+i t y_{0}+o x_{0}}\right) p(t) d t=0
$$

after using (26) and (27). The arbitrary character of $p$ shows easily that the sum vanishes almost everywhere in $t$, and hence the algebraic nature of $s=s(t)$ in $E$ is established. Also the equations (27), (28) now hold for every $(x, y)$, provided we use for $a_{i j}$ their values at $\left(x_{0}, y_{0}\right)$. By (29) the converse is evident (it is also well known), and hence Theorem IV is established.

13. Discussion. The elementary examples first mentioned are in agreement with Theorem IV, as we see by (11), (9), (12), (13), and (16). Equation (16) illustrates the possibility that $\Lambda=0$ at certain points, while (9) and (13) show that more than one branch of the algebraic function may be represented.

The lemma does not say that $\psi(V, X)=0$ is a linear homogeneous equation, nor does it say that every solution of the linear homogeneous equation satisfies $\psi(V, X)=0$. Still less could one say that every solution of the linear equation is in the family $\left\{U_{p}\right\}$. These extensions of the lemma are evidently false. In response to some suggestive questions raised by the referee, however; we have shown that there is a linear system $A_{1} \cdot V=0, A_{2} \cdot V=0, \cdots, A_{m} \cdot V=0$ such that each $U_{p}$ of the linearly closed family satisfies the system, and each solution of the system satisfies $\psi(V, X)=0$. If $\psi$ and $V_{p}$ are continuous, then there exists such a system with continuous $\boldsymbol{A}_{\boldsymbol{i}}$. In this case the original differential operator $\psi$ generally admits the local representation

$$
\psi(\boldsymbol{V}, \boldsymbol{X})=f\left[\left(\boldsymbol{A}_{1} \cdot \boldsymbol{V}\right)^{2}+\left(\boldsymbol{A}_{2} \cdot \boldsymbol{V}\right)^{2}+\cdots+\left(\boldsymbol{A}_{m} \cdot \boldsymbol{V}\right)^{2}\right] G(\boldsymbol{V}, \boldsymbol{X})
$$

where $f(t)$ and $G(V, X)$ are continuous, and $f(0)=0$. More precise statements and proof of these assertions will be given elsewhere.

Note added in proof (July 6, 1953). The discussion of item iii, Theorem $\mathrm{I}$, is inadequate in two respects, and should be modified as follows. First, to show that $\lambda(x, y)$ is a measurable function of $y$, consider $\phi f$ for the family $(2 \pi)^{1 / 2} f(x)=e^{-i u x-x^{2}}$. We have $\phi f=T e^{-x^{2}} \lambda(x, y)$ $=F(u, y)$ where, by hypothesis, $F$ is measurable in $y$ for each $u$. Since $e^{-x^{2}} \lambda \in L$ as a function of $x$ for each $y$, we may compute $e^{-x^{2}} \lambda$ in terms of $F$ as Cesàro mean of the inverse Fourier transform on $u$. This shows that $\lambda(x, y)$ is measurable.

Second, redefine $\lambda(x, y)$ in such a way that $\lim _{i \rightarrow 0}\left|S_{\delta}\right|=\mid \lambda(x$, 
$y) \mid$ for every $x$. The new definition modifies $\lambda(x, y)$ in a set of zero $x$ measure, for each $y$, and hence does not alter the integral in $S_{\delta}$ or that in $\phi$. The first remark shows that the proposed chnage is possible, the second, that it is permissible.

Proceeding as in the proof of the Lebesgue theorem on bounded convergence, one shows that $\int \lim \inf f_{n} \leqq \lim$ inf $\int f_{n}$ for uniformly bounded functions. The result is extended to unbounded functions by considering $\left[f_{n}\right]_{k}$, as in the proof of Fatou's theorem. Thus,

$$
\int_{-m}^{m}|\lambda|^{2} d y=\int \lim \inf \left|S_{\delta}\right|^{2} \leqq \lim \inf \int\left|S_{\delta}\right|^{2} \leqq N,
$$

and we let $m \rightarrow \infty$ as before. Hence item iii holds for all $x$, with the new $\lambda$, not only for almost all $x$. The argument of the text overlooks the fact that the $x$ set in which $\lim S_{\delta}=\lambda$ may depend on $y$.

\section{REFERENCES}

1. G. A. Woonton, The effect of an obstacle in the Fresnel field on the distance field of a linear radiator, Journal of Applied Physics vol. 21 (1950) pp. 577-580.

2. E. Hille, Functional analysis and semi-groups, Amer. Math. Soc. Colloquium Publications, vol. 31, 1948. (Chap. 20 especially, but all is relevant.)

3. B. B. Baker and E. T. Copson, The mathematical theory of Huygens' principle, Oxford, 1950, Chap. 1.

University of California, Los Angeles 